\title{
BMJ Open Ventilation management and outcomes in out-of-hospital cardiac arrest: a protocol for a preplanned secondary analysis of the TTM2 trial
}

\author{
Chiara Robba (D) , ${ }^{1,2}$ Niklas Nielsen, ${ }^{3}$ Josef Dankiewicz, ${ }^{4}$ Rafael Badenes (D) , \\ Denise Battaglini (D) , ,2,6 Lorenzo Ball, ${ }^{1,2}$ Iole Brunetti, ${ }^{1}$ \\ Wendel-Garcia Pedro David (1) ,7 Paul Young (D) , ${ }^{8}$ Glenn Eastwood, ${ }^{9}$ \\ Michelle S Chew, ${ }^{10}$ Janus Jakobsen, ${ }^{11}$ Johan Unden, ${ }^{12,13}$ Matthew Thomas, ${ }^{14}$ \\ Michael Joannidis, ${ }^{15}$ Alistair Nichol, ${ }^{16}$ Andreas Lundin, ${ }^{17}$ Jacob Hollenberg, ${ }^{18}$ \\ Gisela Lilja, ${ }^{12}$ Naomi E Hammond (1) , ${ }^{19}$ Manoj Saxena, ${ }^{20}$ Annborn Martin, ${ }^{21}$ \\ Miroslav Solar, ${ }^{22}$ Fabio Silvio Taccone, ${ }^{23}$ Hans A Friberg, ${ }^{24}$ Paolo Pelosi ${ }^{1,25}$
}

To cite: Robba C, Nielsen N, Dankiewicz J, et al. Ventilation management and outcomes in out-of-hospital cardiac arrest: a protocol for a preplanned secondary analysis of the TTM2 trial. BMJ Open 2022;12:e058001. doi:10.1136/ bmjopen-2021-058001

- Prepublication history for this paper is available online. To view these files, please visit the journal online (http://dx.doi org/10.1136/bmjopen-2021058001).

Received 04 0ctober 2021 Accepted 02 February 2022

Check for updates

(C) Author(s) (or their employer(s)) 2022. Re-use permitted under CC BY-NC. No commercial re-use. See rights and permissions. Published by BMJ.

For numbered affiliations see end of article.

Correspondence to

Dr Chiara Robba;

kiarobba@gmail.com

\section{ABSTRACT}

Introduction Mechanical ventilation is a fundamental component in the management of patients post cardiac arrest. However, the ventilator settings and the gasexchange targets used after cardiac arrest may not be optimal to minimise post-anoxic secondary brain injury. Therefore, questions remain regarding the best ventilator management in such patients.

Methods and analysis This is a preplanned analysis of the international randomised controlled trial, targeted hypothermia versus targeted normothermia after outof-hospital cardiac arrest (OHCA)-target temperature management 2 (TTM2). The primary objective is to describe ventilatory settings and gas exchange in patients who required invasive mechanical ventilation and included in the TTM2 trial. Secondary objectives include evaluating the association of ventilator settings and gas-exchange values with 6 months mortality and neurological outcome. Adult patients after an OHCA who were included in the TTM2 trial and who received invasive mechanical ventilation will be eligible for this analysis. Data collected in the TTM2 trial that will be analysed include patients' prehospital characteristics, clinical examination, ventilator settings and arterial blood gases recorded at hospital and intensive care unit (ICU) admission and daily during ICU stay.

Ethics and dissemination The TTM2 study has been approved by the regional ethics committee at Lund University and by all relevant ethics boards in participating countries. No further ethical committee approval is required for this secondary analysis. Data will be disseminated to the scientific community by abstracts and by original articles submitted to peer-reviewed journals. Trial registration number NCT02908308.

\section{INTRODUCTION}

Many studies in patients after cardiac arrest with return of spontaneous circulation (ROSC) have focused on attempting
Strengths and limitations of this study

This is a preplanned subanalysis of the international randomised controlled trial, targeted hypothermia versus targeted normothermia after out-of-hospital cardiac arrest-target temperature management 2, which focuses on ventilatory management.

- Adult patients (18 years of age or older) who experienced a non-traumatic cardiac arrest of a cardiac or unknown cause with return of spontaneous circulation undergoing mechanical ventilation will be included.

- Positive end-expiratory pressure, plateau pressure, mechanical power, driving pressure, arterial blood gases values-as measured in the first 72 hours from hospital admission-will be described and correlated with outcomes.

- Six months' mortality and neurological status will be collected.

to improve survival rates and neurological outcomes. ${ }^{1-4}$ Despite several interventions have been implemented such as targeted temperature management, blood pressure targets, ${ }^{1-4}$ management of cerebral complications, ${ }^{5-7}$ mortality and poor neurological outcome are still common. ${ }^{4-8} \mathrm{~A}$ number of systemic complications, including cardiovascular and respiratory, have been reported and are associated with worse outcomes. ${ }^{78}$ Few studies have reported the characteristics and the influence of ventilator settings such as tidal volume and positive end-expiratory pressure (PEEP) on outcomes of patients after cardiac arrest $^{9} 10$ and mechanical ventilation practices have not been well described to date. In particular, the role of 
driving pressure and mechanical power during mechanical ventilation to determine outcome in patients after cardiac arrest has been poorly investigated. In addition, the optimal gas-exchange targets have not been clearly defined in this population. Hypoxaemia is a well-known cause of secondary brain injury responsible for cerebral ischaemia and poor outcome. ${ }^{11}$ Recent evidence showed possible detrimental effects of hyperoxaemia, yielding generation of reactive oxygen species, overproduction of free radicals and oxidative stress ${ }^{12}$ and being associated with higher mortality. ${ }^{1314}$ However, in the specific population of cardiac arrest patients, the effect of hyperoxaemia on patients' outcome is still unclear. Similarly, evidence regarding the optimal carbon dioxide $\left(\mathrm{CO}_{2}\right)$ targets is limited. ${ }^{15-19}$

We, therefore, preplanned a secondary analysis of the target temperature management 2 (TTM2) trial (clinicaltrials.gov). The primary objective of this study was to describe the ventilator settings and gas-exchange parameters in adults post out-of-hospital cardiac arrest (OHCA) receiving invasive mechanical ventilation. The secondary objective was to evaluate the association of ventilator settings and gas-exchange parameters with 6 months mortality and neurological outcome.

\section{METHODS AND ANALYSIS \\ Study design}

This is a preplanned secondary analysis of a large international multicentre randomised controlled trial, including mechanically ventilated patients post cardiac arrest and 6-month follow-up. This substudy was approved on the 23 February 2017 by the TTM2 steering committee (details available on https://ttm2trial.org/substudy-proposals)

\section{Objectives}

The primary objective is to describe ventilatory management (such as tidal volume, PEEP, driving pressure, respiratory rate, mechanical power, etc) and gas exchange ( $\mathrm{pHa}$, arterial pressure of oxygen $\left(\mathrm{PaO}_{2}\right)$ and arterial pressure of carbon dioxide $\left.\left(\mathrm{PaCO}_{2}\right)\right)$ in patients who required invasive mechanical ventilation after OHCA and were included in the TTM2 trial within the first 72 hours from recruitment. Secondary objectives are to evaluate the association of ventilator settings and gas exchange with 6-month patients' mortality and neurological status ${ }^{16}$ (by the Modified Rankin Scale (mRS)) (figure 1).

\section{Inclusion}

The TTM2 trial included adult patients (18 years of age or older) who experienced a non-traumatic cardiac arrest of a cardiac or unknown cause with ROSC. In particular:

1. OHCA of a presumed cardiac or unknown cause

2. Sustained ROSC defined as 20 min with signs of circulation without the need for chest compressions.

3. Unconsciousness defined as not being able to obey verbal commands (FOUR Score motor response of $<4$ ) and no verbal response to pain after sustained ROSC.

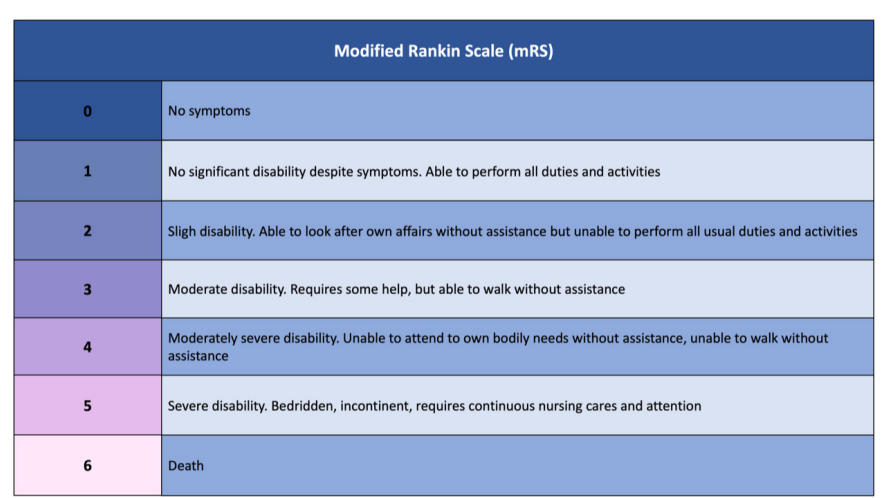

Figure 1 Outcome measure by mRS. mRS, Modified Rankin Scale.

4. Eligible for intensive care without restrictions or limitations

5. Inclusion within $180 \mathrm{~min}$ of ROSC.

Exclusion criteria were:

1. Unwitnessed cardiac arrest with an initial rhythm of asystole.

2. Temperature on admission $<30^{\circ} \mathrm{C}$.

3. On extracorporeal membrane oxygenation prior to ROSC.

4. Obvious or suspected pregnancy.

5. Intracranial bleeding.

6. Severe chronic obstructive pulmonary disorder (COPD) with long-term home oxygen therapy.

For this specific subanalysis, further inclusion criteria will be

- Patients needing mechanical ventilation, and with data available regarding mechanical ventilation settings.

\section{Study procedures and settings}

The protocol of the TTM2 trial has been previously published. ${ }^{15}$ Participants in the TTM2 trial were recruited at multiple sites in several countries. Patients' characteristics were obtained at several timepoints (figure 2). At each of them, objective information of the participants' general physical function and current cardiovascular risk were collected. The subanalysis planned start was December 2021 while planned end will be December 2025, including publications of the manuscripts.

\section{Data collection}

Data were collected at the time of enrolment from the prehospital, from emergency department, during the intensive care unit (ICU) stay, at ICU discharge, at hospital discharge and at follow-up. Clinical, laboratory and background data were collected from hospital records, relatives and ambulance services, and were entered into a web-based electronic case record form (eCRF) by site personnel. For follow-up, all responses were obtained by study personnel from patients or from a proxy (where impaired cognitive capacity prevented patient interview), during a face-to-face visit, by telephone interview or by postal questionnaire. Data from the eCRF of TTM2 used 


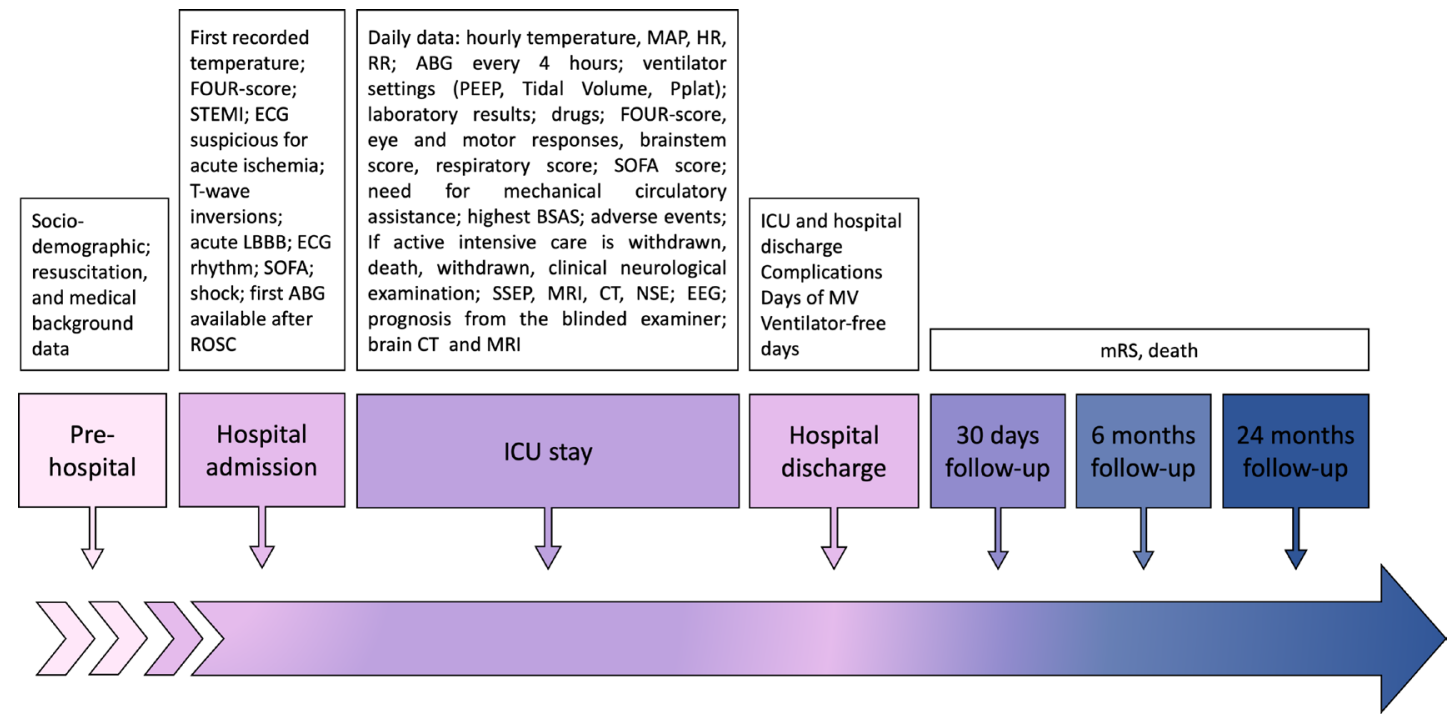

Figure 2 Substudy and study timeline. All collected data at selected timepoints are described. ABG, arterial blood gas; BP, blood pressure; HR, heart rate; ICU, intensive care unit; MAP, mean arterial pressure; mRS, modified Rankin Scale; PEEP, positive end-expiratory pressure; Pplat, plateau pressure; ROSC, return of spontaneous circulation; RR, respiratory rate; SOFA, sequential organ failure assessment.

for this secondary analysis will include patients' demographic characteristics, pre-injury comorbidities, diagnosis, timing of acute events, clinical presentation, timing of intubation, data regarding ventilator settings, daily arterial blood gases, neuromonitoring, ICU complications and outcomes.

Details of the included data are presented in table 1.

\section{Statistical analysis plan}

Patient, ventilator characteristics and arterial blood gases values will be described by means $( \pm \mathrm{SD})$, medians (IQR) and proportions, as appropriate.

The prognostic value of baseline ventilator settingsincluding derived parameters such as driving pressure, mechanical power and compliance-and oxygen $/ \mathrm{CO}_{2}$ markers with mortality endpoints will be evaluated with Cox regression analysis. These survival models will be adjusted for relevant confounders at baseline such as age (years), sex, Charlson Comorbidity Index, time to ROSC (minutes), site where cardiac arrest occurred (home, public place and other), the initial cardiac rhythm found (shockable/non-shockable), bystander CPR (yes/no), witnesses when cardiac arrest occurred (yes/no) and shock or STEMI diagnosis at admission. We will explore the role of currently known threshold of ventilator settings derived in other ICU populations, including plateau pressure, PEEP, driving pressure, ventilatory ratio and mechanical power using relative distribution analysis.

The association between hourly ventilator settings and oxygen $/ \mathrm{CO}_{2}$ markers, and mortality endpoints will be evaluated using linear mixed regression analysis. Here, the longitudinal trajectories of the hourly measured ventilatory settings/blood gases will be compared among survival status at 180 days (dead vs alive) using random intercept in order to account for the longitudinal nature of the data.
The association of baseline ventilator settingsand oxygen $/ \mathrm{CO}_{2}$ markers with mRS outcome will be evaluated with logistic regression analysis. The same set of covariates, as for the survival analysis, will be used. In addition, we will explore new thresholds for the ventilatory settings/blood gases markers on this neurological endpoint, knowing that no specific thresholds along the distribution of these settings have been defined for this outcome.

Statistical analyses will be conducted using Stata V.16.

\section{Data management}

Anonymised data were collected in a web-based eCRF and protected by encryption software and password provided to single users. Each patient was assigned a numeric code generated by the central database. Data were checked for consistency and completeness and monitored by central coordinating centres to ensure high quality of data prior to analysis. The data for this secondary analysis will be securely stored at the University of Genova; all procedures will comply with the EU Regulation 2016/679 on the protection of natural persons regarding personal data processing and movement.

\section{ETHICS AND DISSEMINATION \\ Ethics}

For the TTM2 study, an ethics application (2015/228) was approved by the regional ethics committee at Lund University. Ethics applications was submitted to all relevant ethics boards in every country participating. Each national coordinator (NC)/principal investigator (PI) notified the relevant ethics committee, in compliance with the local legislation and rules. The NCs facilitated this process. The approval of the protocol (if required by local authorities) was obtained before any participant was enrolled. The PI and steering 
Table 1 Timepoints of data collection

\begin{tabular}{|c|c|c|c|c|c|c|}
\hline \multirow[b]{2}{*}{ Patient characteristics } & \multirow[b]{2}{*}{ Data } & \multicolumn{5}{|c|}{ Timepoints } \\
\hline & & $\begin{array}{l}\text { Hospital } \\
\text { stay }\end{array}$ & Daily & $\begin{array}{l}\text { Hospital } \\
\text { discharge }\end{array}$ & 30 days & 6 months \\
\hline \multirow{3}{*}{ Socio-demographic } & Age & $\mathrm{X}$ & & & & \\
\hline & Sex & $\mathrm{x}$ & & & & \\
\hline & Height & $\mathrm{x}$ & & & & \\
\hline \multirow[t]{3}{*}{ Medical background } & Pre-arrest neurological function & $\mathrm{x}$ & & & & \\
\hline & Charlson Comorbidity Index & $x$ & & & & \\
\hline & $\begin{array}{l}\text { Measurement of pre-arrest Clinical Frailty Score } \\
(1-9)\end{array}$ & $x$ & & & & \\
\hline Hospital admission & $\begin{array}{l}\text { SOFA score on admission (without neurological } \\
\text { subscore) }\end{array}$ & $x$ & & & & \\
\hline \multirow{7}{*}{ ICU data } & Arterial blood gases (every 4 hours) & $x$ & $x$ & & & \\
\hline & Haemodynamic (mean arterial pressure and HR) & $x$ & $\mathrm{x}$ & & & \\
\hline & Ventilator settings: PEEP, tidal volume, Pplat & $\mathrm{x}$ & $\mathrm{x}$ & & & \\
\hline & $\begin{array}{l}\text { FOUR Score: eye response, motor response, } \\
\text { brainstem score and respiratory score }\end{array}$ & $x$ & $x$ & & & \\
\hline & SOFA score (without neurological subscore) & $\mathrm{x}$ & $\mathrm{x}$ & & & \\
\hline & Adverse events & $x$ & $x$ & & & \\
\hline & $\begin{array}{l}\text { If trial intervention has been discontinued, time } \\
\text { of discontinuation and specified reason }\end{array}$ & $x$ & $\mathrm{x}$ & & & \\
\hline \multirow[t]{5}{*}{ Post-ICU data } & ICU discharge & & & $\mathrm{x}$ & & \\
\hline & Hospital discharge & & & $\mathrm{x}$ & & \\
\hline & Complications & & & $\mathrm{x}$ & & \\
\hline & Days of mechanical ventilation & & & $\mathrm{x}$ & & \\
\hline & Ventilator-free days & & & $x$ & & \\
\hline \multirow[t]{2}{*}{ Follow-up } & $\mathrm{mRS}$ & & & & $x$ & $x$ \\
\hline & Death & & & & $X$ & $x$ \\
\hline
\end{tabular}

ABG, arterial blood gas; HR, heart rate; ICU, intensive care unit; mRS, Modified Rankin Scale ; PEEP, positive end-expiratory pressure; Pplat, plateau pressure; ROSC, return of spontaneous circulation; SOFA, sequential organ failure assessment.

committee ensured that this study has been conducted in full conformity with the Declaration of Helsinki and good clinical practices. No further ethical approval was planned for this subanalysis as the use of data for substudies was specifically declared in the primary protocol.

\section{Lack of capacity and delayed consent}

For the TTM2 study, informed consent was obtained from patients with no lack of capacity. For patients not able to provide informed consent at the time of recruitment, the responsible clinical/research staff acted as consulter and consent eligible patients after discussion with the next of kin. If the patient had a power of attorney or a legal tutor, he/she acted as consultee and was asked to consent/decline participation to the study on legal behalf of the patient. At follow-up, patients who had regained capacity were asked to provide informed consent and had the possibility to: 
- Provide informed consent for the acute data and follow-up.

- Deny research participation and request destruction of acute data collected.

Consent also included the publication of data for subanalysis; therefore, no further consent forms/applications were requested.

\section{Dissemination}

Results from this study will be disseminated to the scientific community by abstracts, by presentations and by original articles submitted to peer-reviewed journals.

\section{Publication and data sharing policy \\ Publication and authorship}

After the publication of the main papers, data will be made available to the scientific community by means of abstract by scientific papers submitted to peer-reviewed journals. Authorship of the main manuscript will follow the ICMJE recommendations that base authorship on the following four criteria:

- Substantial contributions to the conception or design of the work, or the acquisition, analysis or interpretation of data for the work.

- Drafting the work or revising it critically for important intellectual content.

- Final approval of the version to be published.

- Agreement to be accountable for all aspects of the work in ensuring that questions related to the accuracy or integrity of any part of the work are appropriately investigated and resolved.

A writing committee composed by some members of the core steering committee and biostatisticians and the responsible for the subanalysis will be authors of the manuscript, as for rules predefined by the steering committee of the TTM2 for the primary analysis. CR will be first and PP last author of the manuscripts generated from the subanalysis.

\section{PATIENT AND PUBLIC INVOLVEMENT}

Patient and public were not involved in the design, or conduct, or reporting, or dissemination plans of the research.

\section{DISCUSSION}

This subanalysis of the TTM2 study is designed with the aim to obtain a detailed description of the ventilator management of patients after OHCA.

Post-cardiac arrest syndrome is associated with high morbidity and mortality, which is related not only to a poor neurological outcome but also to respiratory and cardiovascular dysfunctions. ${ }^{16}$ Mechanical ventilation has the goal to maintain an appropriate level of $\mathrm{PaO}_{2}$ and $\mathrm{PaCO}_{2}$. However, the pathophysiology of cardiac arrest and its systemic effects, as well as the relationship between arterial blood gases targets and outcome is still unclear. ${ }^{16}$
Hypoxia has been largely recognised as a major cause of secondary brain injury; recently, also hyperoxia has shown to have potential detrimental effects on patients' outcome. ${ }^{17-27}$

Similarly, hypercapnia can cause cerebral vasodilation and increase intracranial pressure and should, therefore, be avoided, but hypocapnia and cerebral vasoconstriction can lead to cerebral ischaemia. ${ }^{16}$ Normocapnia seems to be associated with good neurological outcome. ${ }^{22} 23$ However, mild hypercapnia has been suggested by some authors to optimise cerebral blood flow and improve survivals. ${ }^{24}$ In summary, optimal ventilator targets are unclear in this group of patients.

Recent literature has highlighted the importance of protective ventilation in patients with acute respiratory distress syndrome (ARDS) and non-ARDS. ${ }^{19}{ }^{25-29}$ However, although these strategies have been suggested also in patients post cardiac arrest, ${ }^{30}$ high PEEP or recruitment manoeuvres may increase intrathoracic pressure and consequently reduce jugular outflow, which can further aggravate cerebral oedema; similarly, low tidal volume and permissive hypercapnia may be detrimental in this group of patients. ${ }^{31}$ Moreover, the role of mechanical power and driving pressure to optimise ventilator settings and improve outcomes has not been investigated so far in this population, but recent evidence suggests the importance of these parameters as integrated physiologic variables potentially associated with ventilator-induced lung injury and worse outcome. ${ }^{32-34}$

The results from this substudy will, therefore, allow to clarify the current status of the ventilator management of these patients, and to discriminate the effects of tidal volume, mechanical power, PEEP and driving pressure on outcomes in patients post cardiac arrest, offering the unique opportunity to globally uniform clinical guidelines regarding ventilator strategies in this group of patients.

\section{Author affiliations}

${ }^{1}$ Department of Anesthesia and Critical Care, San Martino Policlinico Hospital, IRCCS for Oncology and Neuroscience, Genoa, Italy

${ }^{2}$ Dipartimento di Scienze Chirurgiche e Diagnostiche, University of Genoa, Genoa, Italy

${ }^{3}$ Department of Clinical Sciences Lund, Anaesthesia and Intensive Care and Clinical Sciences Helsingborg, Helsingborg Hospital, Lund University, Lund,

Sweden

${ }^{4}$ Department of Clinical Sciences Lund, Cardiology, Skåne University Hospital,Lund University, Lund, Lund, UK

${ }^{5}$ Department of Anesthesiology and Surgical-Trauma Intensive Care, Hospital Clinic Universitari de València, Universitat de València, Valencia, Spain

${ }^{6}$ Department of Medicine, University of Barcelona, Barcelona, Spain, Genoa, Italy

${ }^{7}$ Institute of Intensive Care Medicine, Zurich, Switzerland, University Hospital of

Zürich, Zürich, Switzerland

${ }^{8}$ Department of Intensive Care, Wellington Hospital, Wellington, New Zealand

${ }^{9}$ Department of Intensive Care, Faculty of Health, Deakin University, Burwood, Victoria, Australia

${ }^{10}$ Department of Anaesthesia and Intensive Care, Biomedical and Clinical Sciences, Linköping University, Linköping, Sweden

${ }^{11}$ Copenhagen Trial Unit, Centre for Clinical Intervention Research, Copenhagen University Hospital, Copenhagen, UK

${ }^{12}$ Department of Clinical Sciences, Lund University, Lund, Sweden

${ }^{13}$ Department of Operation and Intensive Care, Hallands Hospital Halmstad, Halland, Sweden 
${ }^{14}$ Department of Anaesthesia, University Hospitals Bristol NHS Foundation Trust, Bristol, UK

${ }^{15}$ Division of Intensive Care and Emergency Medicine, Deptartment of Medicine, Medizinische Universität Innsbruck, Innsbruck, Austria

${ }^{16}$ Monash University, Melbourne, Victoria, Australia, Melbourne, Ireland

${ }^{17}$ Department of Anaesthesiology and Intensive Care Medicine, University of Gothenburg, Gothenburg, Sweden

${ }^{18}$ Department of Medicine, Center for Resuscitation Science, Karolinska Institutet,

Solna, Sweden

${ }^{19}$ Department of Critical Care, George Institute for Global Health, Newtown, New South Wales, Australia

${ }^{20}$ St George Hospital, Sydney, New South Wales, Australia

${ }^{21}$ Department of Clinical Medicine, Anaesthesiology and Intensive Care, Lund University, Lund, Sweden

${ }^{22}$ Department of Internal Medicine, Faculty of Medicine in Hradec Králové, Charles University, Prague, Czech Republic

${ }^{23}$ Department of Intensive Care Medicine, Hopital Erasme, Université Libre de Bruxelles, Brussels, Belgium

${ }^{24}$ Department of of Clinical Sciences Lund, Lund University, Lund, Sweden

${ }^{25}$ Dipartimento di Scienze Chirurgiche e Diagnostiche, Università degli Studi di Genova, Genoa, Italy

Twitter Chiara Robba @chiara_robba and Naomi E Hammond @naomihammond Contributors CR drafted the manuscript and planned the methodology and the outcomes. PP and NN revised the manuscript and supervised the methodology and outcomes. DB prepared figures and tables, helped in the revision and methodology, and approved the final version. JD, RB, LB, IB, W-GPD, PY, GE, MSC, JJ, JU, MT, MJ, AN, AL, JH, GL, NEH, MS, AM, MS, FST and HAF helped in the revision and methodology and approved the final version.

Funding The authors have not declared a specific grant for this research from any funding agency in the public, commercial or not-for-profit sectors.

Competing interests None declared.

Patient and public involvement Patients and/or the public were not involved in the design, or conduct, or reporting, or dissemination plans of this research.

Patient consent for publication Consent obtained from parent(s)/guardian(s)

Provenance and peer review Not commissioned; externally peer reviewed.

Open access This is an open access article distributed in accordance with the Creative Commons Attribution Non Commercial (CC BY-NC 4.0) license, which permits others to distribute, remix, adapt, build upon this work non-commercially, and license their derivative works on different terms, provided the original work is properly cited, appropriate credit is given, any changes made indicated, and the use is non-commercial. See: http://creativecommons.org/licenses/by-nc/4.0/.

\section{ORCID iDs}

Chiara Robba http://orcid.org/0000-0003-1628-3845

Rafael Badenes http://orcid.org/0000-0001-7017-0150

Denise Battaglini http://orcid.org/0000-0002-6895-6442

Wendel-Garcia Pedro David http://orcid.org/0000-0001-7775-3279

Paul Young http://orcid.org/0000-0002-3428-3083

Naomi E Hammond http://orcid.org/0000-0002-6559-7747

\section{REFERENCES}

1 Kim Y-M, Yim H-W, Jeong S-H, et al. Does therapeutic hypothermia benefit adult cardiac arrest patients presenting with nonshockable initial rhythms?: a systematic review and meta-analysis of randomized and non-randomized studies. Resuscitation 2012;83:188-96.

2 Lundbye JB, Rai M, Ramu B, et al. Therapeutic hypothermia is associated with improved neurologic outcome and survival in cardiac arrest survivors of non-shockable rhythms. Resuscitation 2012;83:202-7.

3 Peberdy MA, Callaway CW, Neumar RW, et al. Part 9: postcardiac arrest care: 2010 American heart association guidelines for cardiopulmonary resuscitation and emergency cardiovascular care. Circulation 2010;122:S768-86.

4 Nielsen N, Wetterslev J, Cronberg T, et al. Targeted temperature management at $33^{\circ} \mathrm{C}$ versus $36^{\circ} \mathrm{C}$ after cardiac arrest. $N$ Engl $\mathrm{J}$ Med 2013;369:2197-206.
5 Mentzelopoulos SD, Malachias S, Chamos C, et al. Vasopressin, steroids, and epinephrine and neurologically favorable survival after in-hospital cardiac arrest. JAMA 2013;310:270.

6 Morrison LJ, Neumar RW, Zimmerman JL, et al. Strategies for improving survival after in-hospital cardiac arrest in the United States: 2013 consensus recommendations. Circulation 2013;127:1538-63.

7 Bernard SA, Gray TW, Buist MD, et al. Treatment of comatose survivors of out-of-hospital cardiac arrest with induced hypothermia. N Engl J Med 2002;346:557-63.

8 Hypothermia after Cardiac Arrest Study Group. Mild therapeutic hypothermia to improve the neurologic outcome after cardiac arrest. N Engl J Med 2002;346:549-56

9 Harmon MBA, van Meenen DMP, van der Veen ALIP, et al. Practice of mechanical ventilation in cardiac arrest patients and effects of targeted temperature management: a substudy of the targeted temperature management trial. Resuscitation 2018;129:29-36.

10 Sutherasan Y, Peñuelas O, Muriel A, et al. Management and outcome of mechanically ventilated patients after cardiac arrest. Crit Care 2015;19:215.

11 McHugh GS, Engel DC, Butcher I, et al. Prognostic value of secondary insults in traumatic brain injury: results from the impact study. J Neurotrauma 2007;24:287-93.

12 Young PJ, Bailey M, Bellomo R, et al. Conservative or liberal oxygen therapy in adults after cardiac arrest. Resuscitation 2020;157:15-22.

13 Palmer E, Post B, Klapaukh R, et al. The association between supraphysiologic arterial oxygen levels and mortality in critically ill patients. A multicenter observational cohort study. Am J Respir Crit Care Med 2019;200:1373-80.

14 Y-N N, Wang Y-M, Liang B-M. The effect of hyperoxia on mortality in critically ill patients: a systematic review and meta analysis. BMC Pulm Med 2019;19:53.

15 Lilja G, Nielsen N, Ullén S, et al. Protocol for outcome reporting and follow-up in the targeted hypothermia versus targeted normothermia after out-of-hospital cardiac arrest trial (TTM2). Resuscitation 2020;150:104-12.

16 Roberts BW, Kilgannon JH, Chansky ME, et al. Association between Postresuscitation partial pressure of arterial carbon dioxide and neurological outcome in patients with Post-Cardiac arrest syndrome. Circulation 2013;127:2107-13.

17 Koutsoukou A, Perraki H, Raftopoulou A, et al. Respiratory mechanics in brain-damaged patients. Intensive Care Med 2006;32:1947-54.

18 Patel JK, Kataya A, Parikh PB. Association between intra- and post-arrest hyperoxia on mortality in adults with cardiac arrest: a systematic review and meta-analysis. Resuscitation 2018;127:83-8.

19 Robba C, Ortu A, Bilotta F, et al. Extracorporeal membrane oxygenation for adult respiratory distress syndrome in trauma patients. J Trauma Acute Care Surg 2017;82:165-73.

20 Pilcher J, Weatherall M, Shirtcliffe P, et al. The effect of hyperoxia following cardiac arrest - a systematic review and meta-analysis of animal trials. Resuscitation 2012;83:417-22.

21 Wang $\mathrm{C}-\mathrm{H}$, Chang $\mathrm{W}-\mathrm{T}$, Huang $\mathrm{C}-\mathrm{H}$, et al. The effect of hyperoxia on survival following adult cardiac arrest: a systematic review and meta-analysis of observational studies. Resuscitation 2014;85:1142-8.

22 Roberts BW, Kilgannon J, Chansky ME, et al. Association between initial prescribed minute ventilation and post-resuscitation partial pressure of arterial carbon dioxide in patients with post-cardiac arrest syndrome. Ann Intensive Care 2014;4:9.

23 Eastwood GM, Nichol A. Optimal ventilator settings after return of spontaneous circulation. Curr Opin Crit Care 2020;26:251-8.

24 Eastwood GM, Young PJ, Bellomo R. The impact of oxygen and carbon dioxide management on outcome after cardiac arrest. Curr Opin Crit Care 2014;20:266-72.

25 Karalapillai D, Weinberg L, Peyton P, et al. Effect of intraoperative low tidal volume vs conventional tidal volume on postoperative pulmonary complications in patients undergoing major surgery. JAMA 2020;324:848.

26 Serpa Neto A, Simonis FD, Barbas CSV, et al. Association between tidal volume size, duration of ventilation, and sedation needs in patients without acute respiratory distress syndrome: an individual patient data meta-analysis. Intensive Care Med 2014;40:950-7.

27 Caricato A, Conti G, Della Corte F, et al. Effects of PEEP on the intracranial system of patients with head injury and subarachnoid hemorrhage: the role of respiratory system compliance. J Trauma 2005;58:571-6.

28 Tejerina E, Pelosi P, Muriel A, et al. Association between ventilatory settings and development of acute respiratory distress syndrome in mechanically ventilated patients due to brain injury. J Crit Care 2017;38:341-5. 
29 Neto AS, Barbas CSV, Simonis FD, et al. Epidemiological characteristics, practice of ventilation, and clinical outcome in patients at risk of acute respiratory distress syndrome in intensive care units from 16 countries (PRoVENT): an international, multicentre, prospective study. Lancet Respir Med 2016;4:882-93.

30 Nolan JP, Sandroni C, Böttiger BW, et al. European resuscitation Council and European Society of intensive care medicine guidelines 2021: post-resuscitation care. Resuscitation 2021;161:220-69.

31 Writing Group for the PReVENT Investigators, Simonis FD, Serpa Neto A, et al. Effect of a low vs intermediate tidal volume strategy on
Ventilator-Free days in intensive care unit patients without ARDS: a randomized clinical trial. JAMA 2018;320:320.

32 Serpa Neto A, Deliberato RO, Johnson AEW, et al. Mechanical power of ventilation is associated with mortality in critically ill patients: an analysis of patients in two observational cohorts. Intensive Care Med 2018;44:1914-22.

33 Toufen Junior C, De Santis Santiago RR, Hirota AS, et al. Driving pressure and long-term outcomes in moderate/severe acute respiratory distress syndrome. Ann Intensive Care 2018;8:119.

34 Tonetti T, Vasques F, Rapetti F, et al. Driving pressure and mechanical power: new targets for VILI prevention. Ann Transl Med 2017;5:286. 